\title{
ESTIMATIVA DE INCERTEZA EM ENSAIOS DE RUÍDO DE IMPACTO: POSIÇÃO DOS EQUIPAMENTOS EM MEDIÇÕES EM CAMPO
}

\section{UNCERTAINTY ESTIMATIONFOR IMPACT NOISE TESTS: EQUIPMENT POSITION INFIELDMEASUREMENTS}

\author{
Maria Fernanda de Oliveira Nunes ${ }^{1}$ \\ Universidade de Caxias do Sul, Laboratório de Tecnologia Construtiva, Caxias do Sul, RS, Brasil, mfonunes@ucs.br \\ Vanessa Jorge 2 \\ Universidade de Caxias do Sul, Laboratório de Tecnologia Construtiva, Caxias do Sul, RS, Brasil, nessa.arq08@yahoo.com.br \\ Daniel Tregnago Pagnussat ${ }^{3}$ \\ Universidade de Caxias do Sul, Laboratório de Tecnologia Construtiva, Caxias do Sul, RS, Brasil, dtpagnussat@ucs.br
}

\begin{abstract}
Resumo
A precisão metrológica em ensaios em campo de acústica de edifícios é uma questão atual, com necessidade ampla discussão sobre as variáveis envolvidas que podem gerar dúvidas nos resultados. Nesse contexto, este trabalho analisa as incertezas de medições de ruído de impacto em campo. O foco do trabalho é a análise da influência do posicionamento dos equipamentos. As medições foram realizadas conforme as normas ISO 140-7 e ISO 140-14 em duas salas sobrepostas, separadas por uma laje de concreto maciço de $10 \mathrm{~cm}$ de espessura. Foram consideradas dois grupos de variações no posicionamento da máquina de impactos e do microfone: distâncias mínimas das paredes definidas nas normas ISO e pontos centralizados no piso. As variações no posicionamento dos equipamentos geraram 64 resultados de níveis sonoros na sala de recepção, divididos em quatro grupos de resultados, a partir das medições na faixa de frequência entre 100 e $3.150 \mathrm{~Hz}$. Para as análises foram utilizados os métodos da norma ISO 12999-1 e do Guia RELACRE/Portugal que expressam de formas diferentes as incertezas de medições acústicas em edificações. Os resultados são apresentados na forma de exemplos de aplicação em sistemas pisos ensaiados anteriormente, com diferentes comportamentos acústicos e consequentemente, com valores distintos de níveis de ruído de impacto padronizados ponderados corrigidos pela incerteza estimada. Neste estudo foi evidenciada e quantificada a influência do posicionamento dos equipamentos nos resultados de ensaios de ruído de impacto em campo.
\end{abstract}

Palavras-chave: Ruído de Impacto. Incerteza em Medições. Acústica de Edifícios.

\begin{abstract}
The metrological accuracy on acoustic field measurements of buildings is a current issue that needs extensive discussion due to the fact that the variables involved may challenge their results. In this sense, this paper analyzes the uncertainty of field measurements of impact noise. The focus of the study is the analysis of the influence of equipment positioning. The measurements were performed according to ISO 140-7 and ISO 140-14 standards in two rooms, one above the other, separated by a $10 \mathrm{~cm}$ massive concrete slab. Two groups with different positions of the tapping machine and the microphone were considered: minimum distance of the walls defined by ISO standards and centralized points on the floor. The variations in the positioning of the equipment generated 64 results of sound levels in the reception room, divided into four groups of results, from measurements in the frequency range between 100 and 3,150 Hz. The methods used were based on ISO $12999-1$ standard and RELACRE/Portugal Guide, which express the uncertainties of acoustic measurements in buildings in different ways. The results are presented as examples of application of previously tested floor systems with different acoustic behaviors and, consequently, with different weighted normalized impact sound pressure level of the measured values corrected by the estimated uncertainty. In this study, it was demonstrated and quantified the influence of equipment positioning on the results of field measurements of impact noise in floor systems.
\end{abstract}

Keywords: Impact Noise. Uncertainty in Measurements. Building Acoustics.

How to cite this article:

NUNES, Maria Fernanda de Oliveira; JORGE, Vanessa; PAGNUSSAT, Daniel Tregnago. Estimativa de incerteza em ensaios de ruído de impacto: posição dos equipamentos em medições em campo. PARC Pesquisa em Arquitetura e Construção, Campinas, v. 5, n. 2, p. 22-30, jul./dez. 2014 


\section{Introdução}

O desempenho acústico de um edifício apresenta variações significativas entre os resultados obtidos em laboratório e em campo, sendo que, em laboratório as condições gerais de instalação e de realização dos ensaios têm um controle mais rigoroso.

Para ensaios de determinação do desempenho acústico em campo, as normas brasileiras NBR 15575 (ASSOCIAÇÃO BRASILEIRA DE NORMAS TÉCNICAS, 2013a, 2013b) remetem às normas ISO 140 (INTERNATIONAL ORGANIZATION FOR STANDARDIZATION, 1998, 2004) para os procedimentos de ensaio e às normas ISO 717 (INTERNATIONAL ORGANIZATION FOR STANDARDIZATION, 2013a, 2013b) para a obtenção de número único através da ponderação de valores medidos por frequência. Nesse sentido, a área da acústica de edificações passa por um momento de revisão de normas para ensaios de acústica, especialmente com a entrada em vigor da primeira norma para estimativa de incertezas de medições acústicas, a ISO 12999-1 (INTERNATIONAL ORGANIZATION FOR STANDARDIZATION, 2014), que também acarretará alterações na norma ISO 717 (DODD et al., 2013; MAHN; PEARSE, 2012).

A principal alteração se deve à exigência de se incorporar o método de avaliação de incertezas descrito no guia ISO/IEG Guide 98-3 para a Expressão da Incerteza de Medição que constitui o documento padrão para a avaliação da incerteza de medição em metrologia (INTERNATIONAL ORGANIZATION FOR STANDARDIZATION, 2008).

Nesse contexto, este trabalho analisa comparativamente as incertezas de medições de ruído de impacto em campo, considerando-se a influência de variações no posicionamento dos equipamentos no plano horizontal: proximidade das paredes e proximidade do centro geométrico do piso.

\section{Incerteza em acústica de edifícios}

O resultado de um ensaio pode ser afetado por diversos fatores e, no caso específico das medições acústicas em edifícios, o isolamento de um sistema construtivo pode apresentar significativas variações decorrentes, por exemplo, da execução da obra (CRAIK, 1989; ÖQVIST; LJUNGGREN; ÅGREN, 2012) e da operação dos equipamentos (GERRETSEN, 2007).

A norma ISO/IEC Guide 98-3 (INTERNATIONAL ORGANIZATION FOR STANDARDIZATION, 2008), considera que a estimativa da incerteza de medições acústicas é geralmente constituída por vários componentes que podem ser agrupados em duas categorias de acordo com o método utilizado para calcular os valores numéricos. Segundo a ISO/IEC Guide 98-3, a avaliação do Tipo A é baseada num método de análise estatística válida para o tratamento de dados como o cálculo do desvio padrão da média de uma série de observações independentes ou utilizando o método dos mínimos quadrados para ajustar uma curva a dados. A avaliação da incerteza padrão do Tipo B é, geralmente, baseada em julgamento científico usando toda a informação relevante disponível de especificações do fabricante, os dados fornecidos na calibração e outros relatórios, dados de medições anteriores ou conhecimento geral, o comportamento e as propriedades dos materiais e instrumentos relevantes.

A incerteza de medições acústicas de ruído de impacto em campo foi estudada por Taveira (2012) em 22 ambientes diferentes, com análises dos parâmetros medidos na sala de recepção L2 (nível de pressão sonora de impacto), T30 (tempo de reverberação), LB2 (nível de ruído de fundo), classe de exatidão do analisador sonoro e resultados de calibração do calibrador. A autora concluiu que, para medições em campo de ruído de impacto, o parâmetro L2 é o mais relevante para a estimativa de incerteza e o valor obtido de incerteza

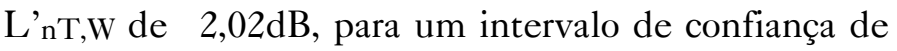
$95 \%$.

A composição espectral do ruído medido também pode causar incertezas específicas e, em especial, as medições em baixas frequências podem estar sujeitas a incertezas devido a ondas estacionárias, elevados níveis de ruído de fundo e vibrações na estrutura transmitidas pelo solo. Por outro lado, os resultados das medições de altas frequências podem revelar incertezas devido à direcionalidade da fonte, alterações na umidade relativa do ar e reflexões de superfícies de dimensões reduzidas dentro dos ambientes de medição (PETERS, 2013). Nesse sentido, Hagberg e Thorsson (2010) ponderam que nas medições de ruído de impacto em sistemas de piso com lajes de concreto, a influência das baixas frequências não é tão importante se comparada à influência nos sistemas de pisos leves.

Carvalho (2013) encontrou maiores variações em resultados de ruído de impacto em laboratório nas frequências a baixo de $100 \mathrm{~Hz}$, que são minimizadas na maioria das situações em que a sala de recepção é menor ou com maior absorção que a sala de emissão. A autor indica que as incertezas podem ser reduzidas com cuidados especiais nas frequências abaixo de $250 \mathrm{~Hz}$, realizando-se um mínimo de seis medições, uniformemente dispersas dentro da sala e que valores extremos ou não representativos devem ser eliminados 
a partir de testes estatísticos. Outro cuidado a ser tomado é em relação a difusibilidade do som na sala de recepção, que deve ser ajustada com o aumento da absorção e a presença de elementos dispersos como, por exemplo, móveis.

Wittstock (2005) ressalta a relevância de se incluir a estimativa de incertezas em medições em campo que devem ser realizadas em condições mais rigorosas de repetibilidade, se a influência do instrumento de medição for negligenciada. O mesmo autor afirma que o desvio padrão para estas medições pode ser descrito, por exemplo, por valores determinados a partir de medições comparativas, desde que a situação seja isenta de dificuldades específicas como, por exemplo, salas muito pequenas ou parcialmente abertas.

Peters (2013) discute as possíveis causas de incertezas em medições acústicas e aponta diferentes causas a serem consideradas, que são associadas à fonte de ruído, ao meio de transmissão, posição do microfone e a sonoridade do ambiente. $\mathrm{O}$ autor considera ainda que a mais relevante fonte de incerteza é a fonte sonora e sua operação, sendo importante considerar: o conteúdo espectral do som emitido, a natureza da fonte sonora (pontual, linear, área), estado de manutenção do equipamento, proximidade de superfícies e variações nos ciclos de operação dos equipamentos.

\section{Norma ISO 12999-1 - Determinação e aplicação de incertezas em acústica de edifícios}

A norma especifica a avaliação detalhada da incerteza, a determinação das incertezas para ensaios inter-laboratoriais e a aplicação das incertezas.

Segundo a ISO 12999-1 (INTERNATIONAL ORGANIZATION

FOR

STANDARDIZATION, 2014), as incertezas devem ser preferencialmente determinadas segundo critérios da ISO/IEC Guide 98-3, e o conceito de incerteza está definido como: parâmetro associado aos resultados de medições, os quais são caracterizados pela dispersão de valores que podem ser razoavelmente atribuídos ao mensurando (objeto quantificável sujeito a medição).

A incerteza expandida $U$ é obtida pelo produto do fator de abrangência $k$ e da incerteza padrão $u$, determinada conforme a Tabela 1 .

Para fins de estimativas de incerteza, a ISO 129991 (INTERNATIONAL ORGANIZATION FOR
STANDARDIZATION, 2014) admite que os valores de uma medição apresentam distribuição Gaussiana e que o valor mínimo de $\mathrm{k}=1$ deve ser utilizado.

$\mathrm{Na}$ norma são diferenciadas três situações de medições:

- Situação A: para ensaios em laboratório.

- Situação B: para ensaios realizados por equipes diferentes no mesmo local.

- Situação C: para ensaios realizados pela mesma equipe no mesmo local.

Tabela 1 - Incertezas padrão para isolamento ao ruído de impacto em bandas de $1 / 3$ de oitava

\begin{tabular}{|c|c|c|}
\hline Frequência (Hz) & Situação B (dB) & Situação C (dB) \\
\hline 50 & 3,2 & 1,5 \\
\hline 63 & 2,8 & 1,4 \\
\hline 80 & 2,4 & 1,3 \\
\hline 100 & 2,0 & 1,2 \\
\hline 125 & 1,6 & 1,1 \\
\hline 160 & 1,4 & 1,0 \\
\hline 200 & 1,3 & 0,9 \\
\hline 250 & 1,2 & 0,8 \\
\hline 315 & 1,2 & 0,8 \\
\hline 400 & 1,2 & 0,8 \\
\hline 500 & 1,2 & 0,8 \\
\hline 630 & 1,2 & 0,8 \\
\hline 800 & 1,2 & 0,8 \\
\hline 1000 & 1,2 & 0,8 \\
\hline 1250 & 1,3 & 0,8 \\
\hline 1600 & 1,4 & 0,8 \\
\hline 2000 & 1,5 & 0,8 \\
\hline 2500 & 1,7 & 1,0 \\
\hline 3150 & 1,9 & 1,2 \\
\hline 4000 & 2,1 & 1,4 \\
\hline 5000 & 2,3 & 1,6 \\
\hline
\end{tabular}

Fonte: ISO 12999-1:2014 (E)

Atualmente, não existem resultados analisados em condições de repetibilidade para ruído de impacto, por isso, conforme critérios da norma ISO 12999-1 (INTERNATIONAL ORGANIZATION FOR STANDARDIZATION, 2014), são consideradas somente as situações B e $\mathrm{C}$ para determinação da incerteza padrão em ensaios de campo de ruído de impacto. 


\section{Medições de ruído de impacto}

As medições foram realizadas em duas salas sobrepostas, separadas por uma laje de concreto maciço de $10 \mathrm{~cm}$ de espessura, com paredes de alvenaria de tijolos maciços rebocados nas duas faces, com duas de suas paredes externas. A área dos pisos das salas é de $16,2 \mathrm{~m}$ e o volume, de 44,8m .

As salas têm uma janela e uma porta cada, sendo que, na sala de recepção a janela foi vedada com uma chapa de MDF $18 \mathrm{~mm}$, fixada no perímetro da abertura com transpasse de $3 \mathrm{~cm}$ sobre uma camada de EVA.

Os procedimentos de medição seguiram as recomendações na ISO 140-7 (INTERNATIONAL ORGANIZATION FOR STANDARDIZATION, 1998), ISO 140-14 (INTERNATIONAL ORGANIZATION FOR STANDARDIZATION, 2004) e ISO 354 (INTERNATIONAL ORGANIZATION FOR STANDARDIZATION, 2003), com a utilização dos seguintes equipamentos: analisador sonoro B\&K 2270, amplificador de potência $\mathrm{B} \& \mathrm{~K}$, máquina de impactos $\mathrm{B} \& \mathrm{~K}$ e fonte dodecaédrica $\mathrm{B} \& \mathrm{~K}$, com certificados de calibração válidos até junho de 2014. A temperatura e umidade relativa foram monitoradas ao longo de todo o ensaio, a partir de medições com o monitor de estresse térmico Quest Temp 36, configurado para o tempo de aquisição a cada 1 minuto.

Todas as medições foram realizadas no dia 17 de abril de 2014 pelo mesmo operador, sem a presença de precipitações atmosféricas, com temperatura e umidade relativa no interior das salas de $21,5 \mathrm{C}$, com variações de $1,5 \mathrm{C}$, e $80 \%$, com variações de $8,5 \%$, respectivamente.

Foram respeitados os limites mínimos de afastamento de $0,5 \mathrm{~m}$ das superfícies internas, $0,7 \mathrm{~m}$ entre posições de microfone para a determinação das variações de posicionamento dos equipamentos. Desta forma, foram definidos quatro conjuntos a partir das duas variações de posicionamento da máquina de impactos e do microfone, para os quais foram atribuídas identificações específicas denominadas de "Grupos A, B, C e D" (Figura 1), com duas medições em cada ponto, totalizando 128 resultados de L2. Os valores de L'nT,W encontram-se na Tabela 2.

As Figuras 2 a 5 apresentam os resultados por ponto de medição em L' nT para os quatro grupos com as variações nas posições dos equipamentos. Os pontos de medição, identificados nas legendas dos gráficos por "Pos", foram numerados a partir da sequência de execução das medições. Pode-se perceber que nos Grupos A e B (Figuras 2 e 3 ) ocorreu maior distinção nos perfis gráficos. Destacam-se as diferenças entre os valores das posições representadas em preto, para as quais a máquina de impactos estava mais próxima à janela.

Figura 1 - Esquema do posicionamento dos equipamentos na sala de emissão e na sala de recepção
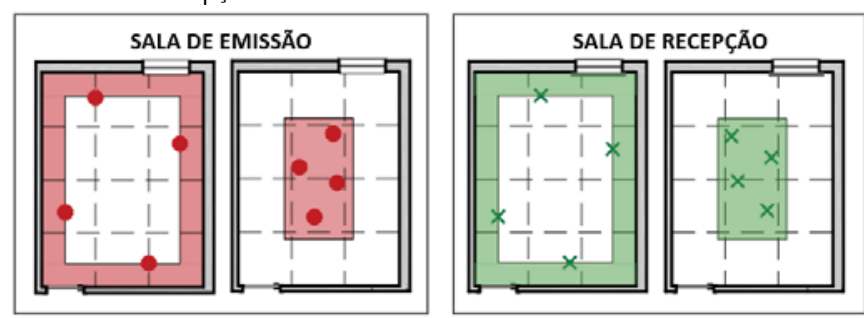

Fonte: os autores

Tabela 2 - Esquema geral e valores em L'nT,w por grupo de medição

\begin{tabular}{|c|c|c|c|c|}
\hline $\begin{array}{l}\text { Grupo de } \\
\text { medição }\end{array}$ & $\begin{array}{c}\text { Esquema } \\
\text { geral da } \\
\text { medição }\end{array}$ & $\begin{array}{l}\text { Posição } \\
\text { máquina de } \\
\text { impactos }\end{array}$ & $\begin{array}{l}\text { Posições } \\
\text { do } \\
\text { microfone }\end{array}$ & $\begin{array}{l}L^{\prime} n T, w \\
\text { (dB) }\end{array}$ \\
\hline Grupo A & . & Próximo à parede & $\begin{array}{l}\text { Próximo à } \\
\text { parede }\end{array}$ & 73 \\
\hline Grupo B &. & Próximo à parede & $\begin{array}{c}\text { Centro da } \\
\text { sala }\end{array}$ & 72 \\
\hline Grupo C & 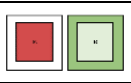 & Centro da sala & $\begin{array}{l}\text { Próximo à } \\
\text { parede }\end{array}$ & 73 \\
\hline Grupo D & ${ }^{*}$ & Centro da sala & $\begin{array}{l}\text { Centro da } \\
\text { sala }\end{array}$ & 72 \\
\hline
\end{tabular}

Fonte: os autores

Para os Grupos C e D, nos quais a máquina de impactos foi posicionada na área central do piso, as maiores variações foram verificadas nas frequências até $200 \mathrm{~Hz}$ (Figuras 4 e 5).

A partir desses resultados, é possível afirmar que o posicionamento central da máquina de impactos reduz as diferenças acarretadas pela presença de portas e janelas dos ambientes de medição.

Figura 2 - Valores em L'nT para fonte e microfone próximos à parede

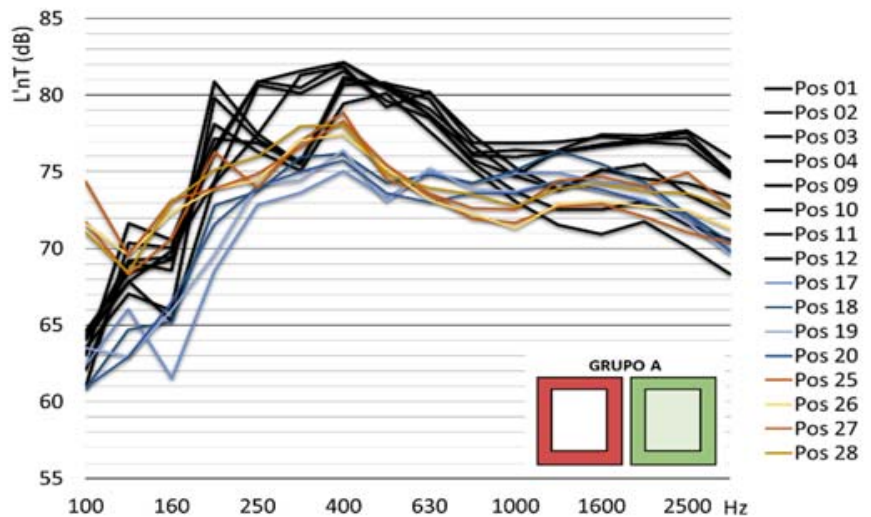

Fonte: os autores 
Figura 3 - Valores em L'nт para fonte próxima à parede e microfone na área central da sala

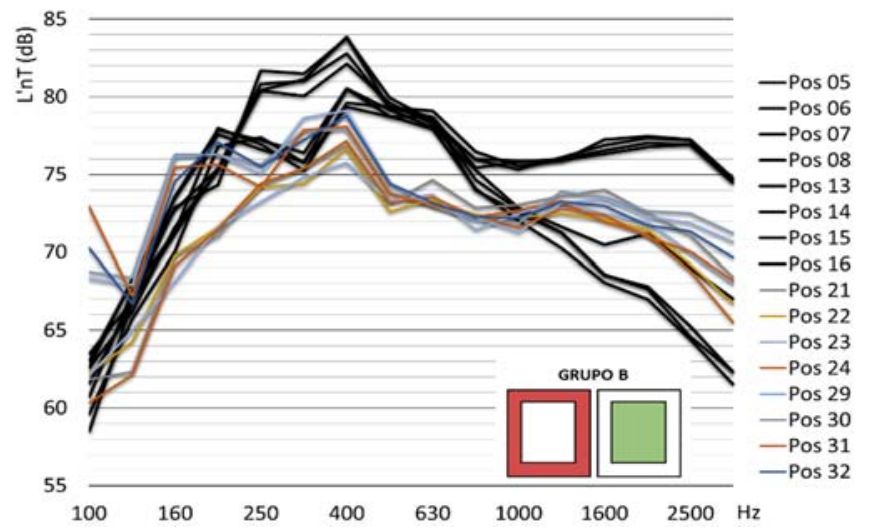

Fonte: os autores

Figura 4 - Valores em L'nт para fonte na área central da sala e microfone próximo à parede

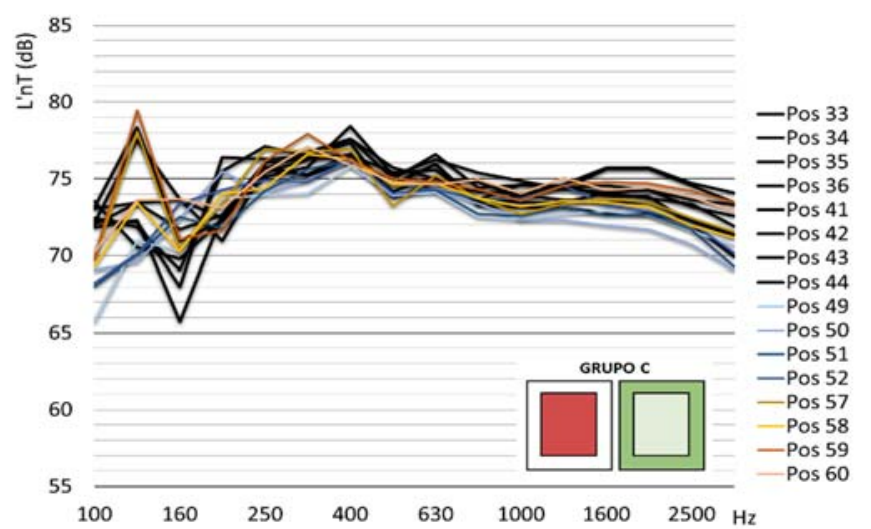

Fonte: os autores

Figura 5 - Valores em L'nт para fonte e microfone na área central da sala

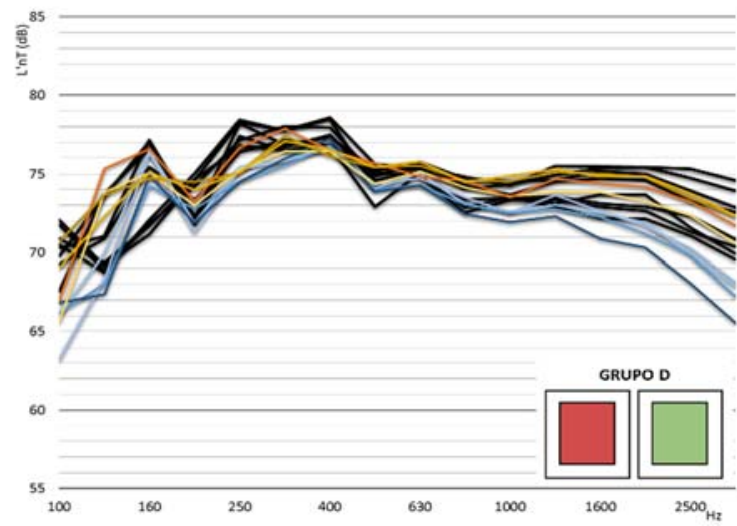

Fonte: os autores

\section{Estimativa da incerteza nas medições}

Foram utilizados critérios estatísticos com o uso do software SPSS 18 nos 64 resultados de L2, agrupados conforme os critérios de posicionamento mencionados.

\section{Critério de rejeição de valores}

Para a identificação dos valores considerados estatisticamente dispersos no conjunto dos resultados utilizou-se o critério de Grubbs, conforme estudos de Michalski, Ferreira e Nabuco (2008) e Michalski (2011).

O teste de Grubbs é utilizado para verificar se as médias obtidas pelos vários laboratórios são compatíveis, com a finalidade de se tomar decisões sobre os valores a serem rejeitados. Considera-se que o menor valor, x1, e o maior valor, $\mathrm{x}_{\mathrm{n}}$, são passíveis de serem rejeitados. Desta forma, o teste de Grubbs é definido por:

$$
G=\frac{\left|x_{i}-\bar{x}\right|}{s}
$$

Onde:

$x_{i}$ é o resultado da i-ésima medição,

$\bar{x}$ a média aritmética do conjunto de valores da amostra, $s$ é o desvio padrão do conjunto de valores.

No total, foram rejeitados 29 valores de L'nT. A maior quantidade de valores rejeitados ocorreu nas frequências abaixo de $400 \mathrm{~Hz}$, sendo que, a $315 \mathrm{~Hz}$ houve a rejeição de seis valores (Tabela 3). Com exceção dos três valores rejeitados na frequência de $125 \mathrm{~Hz}$, resultantes do uso da máquina de impactos na área central da sala, todos os outros 26 valores rejeitados foram obtidos nas medições com a máquina de impactos posicionada à distância mínima da parede.

\section{Desvio padrão}

Os desvios padrão por amostra nos quatro grupos de medição, pelo critério de Grubbs, apresentaram valores maiores nos dois grupos com a máquina de impactos colocada na distância mínima em relação às paredes. Essa diferença é mais significativa nas frequências entre $200 \mathrm{~Hz}$ e $1,25 \mathrm{kHz}$ nos dois grupos, mas no grupo de medições com o microfone posicionado na área central da sala essa diferença se mostrou crescente em relação às maiores frequências (Figura 6).

Segundo Taylor (2012) os valores de um determinado intervalo de resultados, para fins de estimativas de incerteza, são sempre simétricos e relação à média desses resultados. Desta forma, define-se os Grupos C e 
D como mais representativos neste estudo, pois os perfis gráficos gerados com os valores dos desvios apresentam simetria em relação aos valores calculados nas médias.

Tabela 3 - Valores rejeitados pelo critério de Grubbs

\begin{tabular}{cc}
\hline $\begin{array}{c}\text { Frequências com valores } \\
\text { rejeitados (Hz) }\end{array}$ & Quantidade de valores rejeitados \\
\hline 125 & 3 \\
\hline 160 & 2 \\
\hline 200 & 2 \\
\hline 250 & 4 \\
\hline 315 & 6 \\
\hline 400 & 4 \\
\hline $1 \mathrm{k}$ & 2 \\
\hline $1,25 \mathrm{k}$ & 1 \\
\hline $1,6 \mathrm{k}$ & 1 \\
\hline $2 \mathrm{k}$ & 1 \\
\hline $2,5 \mathrm{k}$ & 2 \\
\hline $3,15 \mathrm{k}$ & 1 \\
\hline
\end{tabular}

Fonte: os autores

Figura 6 - Gráfico com as médias L'nт por grupo de posições (eixo vertical esquerdo) e desvios padrão por grupo de posições (eixo vertical direito)

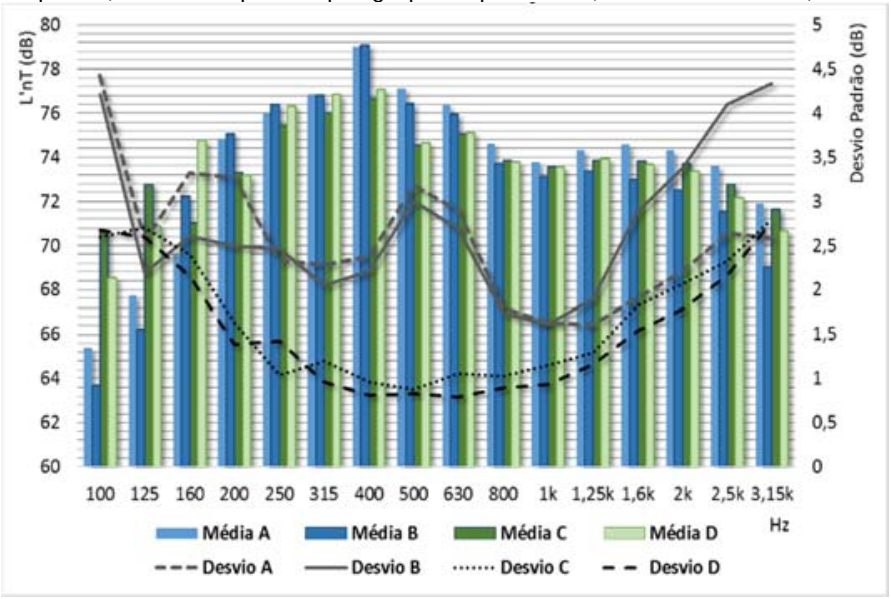

Fonte: os autores

\section{Incertezas estimadas}

Considerando-se que não houve variações significativas na temperatura nem umidade relativa na sala durante as medições e que foi admitido o mesmo tempo de reverberação para todos os resultados, foram definidas como fontes de incerteza o que pode gerar dúvidas decorrentes das possíveis variações de posição dos equipamentos no plano horizontal das salas de emissão e de recepção.

Tendo como base estudos anteriores (MICHALSKI, 2011; ASSOCIAÇÃO DE LABORATÓRIOS ACREDITADOS DE PORTUGAL, 2012), os fatores relacionados ao arredondamento do equipamento e à calibração não foram considerados como fonte de incerteza padrão devido à sua reduzida contribuição na estimativa da incerteza expandida, aproximadamente $0,1 \mathrm{~dB}$.

As medições foram realizadas pela mesma equipe, utilizando os mesmos equipamentos, com a mesma pessoa operando o analisador sonoro e repetidas no mesmo local e, sendo assim, foi definida a Situação $C$ da ISO 12999-1 (INTERNATIONAL ORGANIZATION FOR STANDARDIZATION, 2014) com os valores de incerteza padrão conforme a Tabela 4. Desta forma, a incerteza padrão é o desvio padrão da repetibilidade das medições, considerando-se $\mathrm{k}=1$.

Tabela 4 - Incertezas por frequência para cada grupo de medição

\begin{tabular}{cccccc}
\hline \multirow{2}{*}{$\begin{array}{c}\text { Frequência } \\
(\mathbf{H z})\end{array}$} & \multicolumn{5}{c}{ Incertezas (dB) } \\
\cline { 2 - 6 } & Grupo A & Grupo B & Grupo C & Grupo D & ISO 12999 \\
\hline 100 & 1,14 & 1,09 & 0,71 & 0,73 & 1,2 \\
\hline 125 & 0,70 & 0,62 & 0,73 & 0,71 & 1,1 \\
\hline 160 & 0,88 & 0,71 & 0,67 & 0,61 & 1,0 \\
\hline 200 & 0,87 & 0,69 & 0,50 & 0,45 & 0,9 \\
\hline 250 & 0,65 & 0,68 & 0,39 & 0,46 & 0,8 \\
\hline 315 & 0,64 & 0,59 & 0,42 & 0,38 & 0,8 \\
\hline 400 & 0,66 & 0,62 & 0,38 & 0,35 & 0,8 \\
\hline 500 & 0,85 & 0,81 & 0,36 & 0,36 & 0,8 \\
\hline 630 & 0,78 & 0,73 & 0,39 & 0,35 & 0,8 \\
\hline 800 & 0,53 & 0,52 & 0,39 & 0,37 & 0,8 \\
\hline $1 \mathrm{k}$ & 0,50 & 0,50 & 0,41 & 0,37 & 0,8 \\
\hline $1,25 \mathrm{k}$ & 0,50 & 0,56 & 0,44 & 0,41 & 0,8 \\
\hline $1,6 \mathrm{k}$ & 0,56 & 0,78 & 0,54 & 0,48 & 0,8 \\
\hline $2 \mathrm{k}$ & 0,62 & 0,89 & 0,59 & 0,53 & 0,8 \\
\hline $2,5 \mathrm{k}$ & 0,72 & 1,06 & 0,65 & 0,61 & 1,0 \\
\hline $3,15 \mathrm{k}$ & 0,71 & 1,12 & 0,76 & 0,75 & 1,2 \\
\hline
\end{tabular}

Fonte: os autores

O gráfico da Figura 7 compara os valores de incerteza para os quatro grupos de estudo com os valores definidos pela ISO 12999-1 (INTERNATIONAL ORGANIZATION FOR STANDARDIZATION, 2014). Verifica-se que o posicionamento central da máquina de impactos, resulta em valores mais distantes dos previstos pela ISO 12999-1 (INTERNATIONAL ORGANIZATION FOR STANDARDIZATION, 2014).

Apesar de apresentarem valores menores aos da ISO 12999-1 (INTERNATIONAL ORGANIZATION FOR STANDARDIZATION, 2014), a distribuição das incertezas por frequência apresenta uma similaridade maior ao da ISO 12999-1 (INTERNATIONAL ORGANIZATION FOR STANDARDIZATION, 2014) (Figura 7). Ou seja, as maiores dúvidas em relação 
aos resultados por frequências são esperadas até $200 \mathrm{~Hz}$ e a partir de $2500 \mathrm{~Hz}$.

Figura 7 - Gráfico comparativo das incertezas padrão para cada grupo de medição comparativamente às da ISO 12999-1

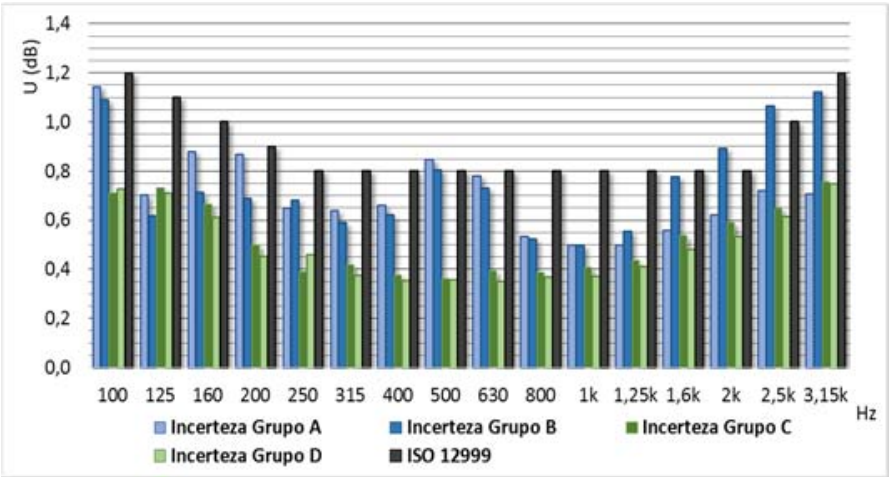

Fonte: os autores

\section{Exemplos de aplicação da estimativa de incerteza}

A aplicação da incerteza de medições acústicas em edificações nos resultados de um ensaio consiste na utilização da variação prevista nos resultados dos níveis de ruído padronizados de impacto L'nT. Avalia-se que as maiores incertezas são estimadas para as frequências abaixo de $200 \mathrm{~Hz}$ e acima de $2000 \mathrm{~Hz}$, e que diferentes sistemas de pisos apresentam diferenças na incerteza expandida (U) e diferentes nos valores de L'nT,W após a aplicação das correções $\left(R_{c}\right)$, em função das diferentes composições espectrais decorrentes de diferenças construtivas.

Nas Figuras 8, 9 e 10 são apresentados exemplos de aplicação das incertezas do Grupo C, Grupo D e da ISO 12999-1 (INTERNATIONAL ORGANIZATION FOR STANDARDIZATION, 2014) nos resultados de três diferentes sistemas de pisos ensaiados previamente. Os três exemplos apresentam diferentes curvas de L'nT e os valores em L'nT,W estão apresentados nos rótulos das barras em vermelho. Os resultados estão expressos conforme a ISO 12999-1 (INTERNATIONAL ORGANIZATION FOR STANDARDIZATION, 2014), com uma casa decimal.

Os resultados em L'nT do sistema de piso 1 apresentam valores por frequência mais equilibrados e o L'nT,W foi de 77,4dB. Os resultados corrigidos (Rc+ e Rc-) decorrentes das estimativas dos ensaios em campo foram de 78 e 76,8 dB, para a localização do microfone próximo as paredes e no centro da sala. Para a estimativa de incerteza conforme critérios da ISO 12999-1 (INTERNATIONAL ORGANIZATION FOR STANDARDIZATION, 2014) os valores corrigidos foram de 78,3 e 76,5 dB (Figura 8).
Figura 8 - Aplicação das correções no sistema de piso 1
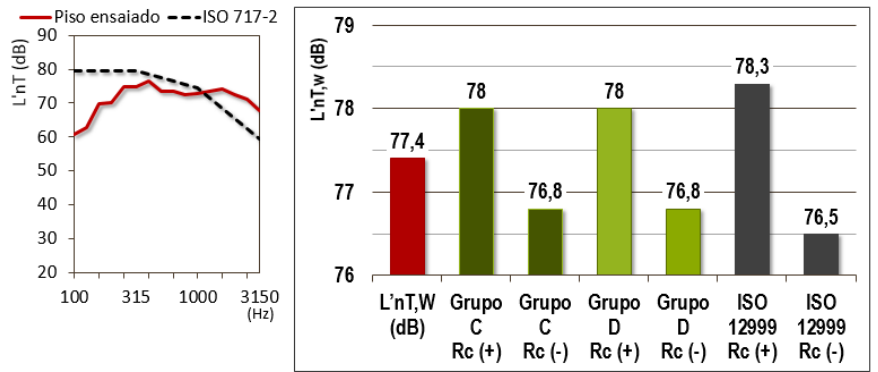

Fonte: os autores

Para o sistema de piso 2 os resultados em L'nT os valores por frequência caracterizaram maior isolamento ao ruído de impacto nas altas frequências, com L' nT,W de 49,1dB. Os resultados corrigidos (Rc+ e Rc-) decorrentes das estimativas dos ensaios em campo foram de 49,6 e 48,6 $\mathrm{dB}$, para a localização do microfone próximo as paredes e no centro da sala. Para a estimativa de incerteza conforme critérios da ISO 12999-1 (INTERNATIONAL ORGANIZATION FOR STANDARDIZATION, 2014) os valores corrigidos foram de 49,9 e 48,3 dB (Figura 9).

Figura 9 - Aplicação das correções no sistema de piso 2
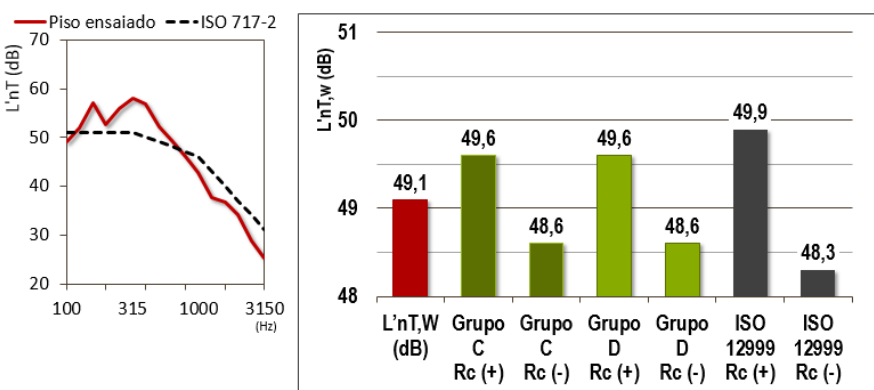

Fonte: os autores

O terceiro sistema de piso analisado foi caracterizado com isolamento acústico deficiente nas altas frequências, com L'nT,W de 74,8dB. Os resultados corrigidos (Rc+e Rc-) decorrentes das estimativas dos ensaios em campo foram de 75,4 e 74,2 dB. Para a estimativa de incerteza conforme critérios da ISO 12999-1 (INTERNATIONAL ORGANIZATION FOR STANDARDIZATION, 2014) os valores corrigidos foram de 75,7 e 73,8 dB (Figura 10).

Cabe salientar que a ISO 12999-1 (INTERNATIONAL ORGANIZATION FOR STANDARDIZATION, 2014) prevê a utilização das bandas centrais de terço de oitava entre $50 \mathrm{~Hz}$ e $5000 \mathrm{~Hz}$, mas como sua publicação ocorreu após a conclusão dos ensaios, optou-se por restringir o intervalo de frequências para a conclusão deste estudo. 
Figura 10 - Aplicação das correções n sistema de piso 3
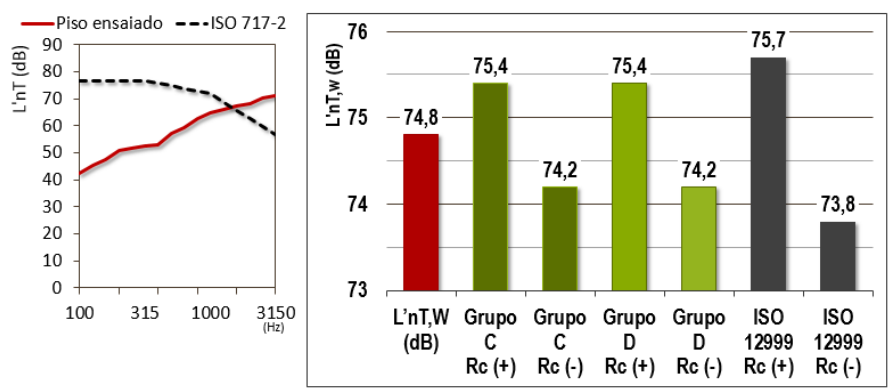

Fonte: os autores

\section{Considerações finais}

Nas medições em campo diversos fatores podem originar dúvidas em relação aos resultados e a minimização da influência desses fatores constitui uma das formas de garantia da qualidade metrológica dos laboratórios, pois sempre que se realiza algum tipo de medição se cometem erros devido a causas diversas.

A partir desse estudo foi verificado que o posicionamento da máquina de impactos próximo às paredes, especialmente nos pontos próximos a janelas, gera níveis de ruído de impacto maiores, comparados aos pontos localizados no centro do piso. Além disso, a distribuição dos valores por frequência não permite uma análise estatística comparável ao método de estimativa de incertezas da norma ISO 12999-1 (INTERNATIONAL ORGANIZATION FOR STANDARDIZATION, 2014). No entanto, não se deve descartar totalmente esses pontos em uma medição em campo, pois a produção do impacto mecânico decorrente do uso do imóvel também pode ocorrer próxima a janelas.

As maiores correções são verificadas com a utilização das incertezas definidas pela ISO 12999-1 (INTERNATIONAL ORGANIZATION FOR STANDARDIZATION, 2014), em especial nos pisos caracterizados por maiores níveis sonoros de impacto medidos nas altas frequências, com valor de correção de
0,9dB. Aparentemente, esse valor pode ser considerado pequeno, mas essa diferença pode alterar a classificação de desempenho de um sistema de piso, considerandose resultados de medição próximos aos limites de classificação.

Os cuidados com a manutenção adequada dos equipamentos, o rigor na aplicação das recomendações das normativas pertinentes e a qualificação da equipe de operadores são assumidamente responsabilidades atribuídas ao laboratório responsável por ensaios em campo. No entanto, equivocadamente, a qualificação da equipe pode ser entendida somente como a capacidade na operação dos equipamentos, mas a capacidade de avaliar corretamente as diferentes situações em campo e de analisar adequadamente as incertezas a serem assumidas nos resultados são de grande relevância para o desenvolvimento dessa qualificação. Nesse caso, com a maior experiência nos processos de medições acústicas em campo, a análise mais crítica e a identificação dos fatores que podem gerar dúvidas e afetar os resultados qualificam e determinam a capacidade de uma equipe e/ou operador.

Neste breve trabalho, foram apresentados resultados de um estudo sobre a influência de diferentes posicionamentos dos equipamentos nos resultados de medições em campo de ruído de impacto em um sistema de piso. As demais variáveis como, por exemplo, fatores comportamentais do operador dos equipamentos, diferenças na calibração dos equipamentos, diferenças no espaço físico e condições atmosféricas foram devidamente controladas e não foram aqui consideradas.

Apesar da entrada em vigor da norma ISO 12999-1 (INTERNATIONAL ORGANIZATION FOR STANDARDIZATION, 2014), as considerações sobre incertezas de medições acústicas em campo ainda podem ser classificadas como complexas e requerem mais análises para a devida identificação das fontes de incerteza mais relevantes.

\section{Referências}

ASSOCIAÇÃO BRASILEIRA DE NORMAS TÉCNICAS. NBR 15575-3 Edifícios Habitacionais - Desempenho. Parte

3: Requisitos para os sistemas de pisos. Rio de Janeiro, 2013a.

NBR 15575-3 Edifícios Habitacionais - Desempenho. Parte 4: Requisitos para os sistemas de vedações verticais internas e externas - SVVIE. Rio de Janeiro, 2013b.

ASSOCIAÇÃO DE LABORATÓRIOS ACREDITADOS DE PORTUGAL. Guia RELACRE 22 - Cálculo de incertezas - Acústica. Lisboa: RELACRE, 2012.

CARVALHO, A. P. O. Reproducibility in interlaboratory impact sound insulation measurements. In:

INTERNATIONAL CONGRESS ON SOUND AND VIBRATION, 13.,Viena, 2013. Proceedings... Viena, 2013. 
CRAIK, R. J. M. The effect of workmanship on sound transmission through buildings: Part 2 structure-borne sound. Applied Acoustics, v. 27, p. 137-145. 1989. http://dx.doi.org/10.1016/0003-682X(89)90006-6

DODD, G.; SCHMID, G.; MARTENS, D. Accuracy and Purpose of Building Insulation Measurements. New Zealand Acoustics, v. 26, n. 1, p. 19-29, 2013.

GERRETSEN, E. Interpretation of uncertainties in acoustic measurements in buildings. Noise Control Engineering Journal, v. 55(1), p. 50-54. 2007. http://dx.doi.org/10.3397/1.2402311

HAGBERG, K.; THORSSON, P. Uncertainties in standard impact sound measurement and evaluation procedure applied to light weight structures. In: INTERNATIONAL CONGRESS ON ACOUSTICS, 20., Sydney, 2010. Proceedings... Sydney, 2010.

INTERNATIONAL ORGANIZATION FOR STANDARDIZATION. ISO 140-7 Acoustics - Measurement of sound insulation in building elements - Part 7 Field measurements of impact sound insulation of floors. Genebra, 1998.

ISO 140-14 Acoustics - Measurement of sound insulation in buildings and of building elements - Guidelines for special situations in the field. Geneva, 2004.

ISO 354 Acoustics -Measurement of sound absorption in a reverberation room. Geneva, 2003.

ISO 717-1 Acoustics - Rating of sound insulation in buildings and of building elements. Part 2: Airborne sound insulation. Geneva, 2013a.

ISO 717-2 Acoustics - Rating of sound insulation in buildings and of building elements. Part 2: Impact sound insulation. Geneva, 2013b.

ISO 12999-1 Acoustics - Determination and application of measurement uncertainties in building acoustics - Part 1: Sound insulation. Geneva, 2014.

ISO/IEC Guide 98-3 Uncertainty of measurement - Part 3: Guide to the expression of uncertainties in measurements (GUM: 1995). Geneva, 2008.

MAHN, J.; PEARSE, J. The Uncertainty of the Proposed Single Number Ratings for Airborne Sound Insulation. Building Acoustics, v. 19, n. 3, p. 145-172, set. 2012. http://dx.doi.org/10.1260/1351-010X.19.3.145

MICHALSKI, R. L. X. N. Metodologias para medição de isolamento sonoro em campo e para expressão da incerteza de medição na avaliação do desempenho acústico de edificações. 2011. 235 f. Tese (Doutorado em Engenharia Mecânica) COPPE - Universidade Federal do Rio de Janeiro, 2011.

MICHALSKI, R. L. X. N.; FERREIRA, D. P. F.; NABUCO, M. Incerteza em Medição em Campo de Isolamento Sonoro Aéreo. In: ENCONTRO DA SOCIEDADE BRASILEIRA DE ACÚSTICA, 22., 2008. Anais... Belo Horizonte, 2008.

ÖQVIST, R., LJUNGGREN, F.; ÅGREN, A. On the uncertainty of building acoustic measurements - Case study of a cross-laminated timber construction. Applied Acoustics, v. 73(9), p. 904-912. 2012.

http://dx.doi.org/10.1016/j.apacoust.2012.03.012

PETERS, R. J. Acoustics and Noise Control. 3 ed. Nova Jersey: Taylor \& Francis, 2013. 386 p.

TAYLOR, J R. Introdução à Análise de Erros: O Estudo de Incertezas em Medições Físicas. 2. ed. Porto Alegre: Bookman, 2012. p. 327.

TAVEIRA, C. S. S. Incerteza na medição do índice de isolamento sonoro a ruído de percussão. 2012.147 f. Dissertação (Mestrado em Engenharia Civil) - Faculdade de Engenharia - Universidade do Porto, Porto, 2012.

WITTSTOCK, V. Uncertainties in building acoustics. In: FORUM ACUSTICUM. 2005, Budapeste. Proceedings... Budapeste: EUROPEAN ACOUSTICS ASSOCIATION, 2005.

\footnotetext{
${ }^{1}$ Maria Fernanda de Oliveira Nunes Arquiteta e Urbanista. Doutora em Engenharia. Endereço postal: Avenida Frederico Segala, 3099, Caxias do Sul, RS, Brasil, CEP 95010-550.

${ }^{2}$ Vanessa Jorge

Graduando em Arquitetura e Urbanismo. Endereço postal: Avenida Frederico Segala, 3099, Caxias do Sul, RS, Brasil, GEP 95010-550.

${ }^{3}$ Daniel Tregnago Pagnussat

Engenheiro Givil. Doutor em Engenharia. Endereço postal: Avenida Frederico Segala, 3099, Caxias do Sul, RS, Brasil, GEP 95010-550.
} 